\title{
Deleterious mutation
}

National Cancer Institute ( $\mathrm{NCl})$

\section{Source}

National Cancer Institute (NCI). Deleterious mutation.

A genetic alteration that increases an individual's susceptibility or predisposition to a certain disease or disorder. When such a variant (or mutation) is inherited, development of symptoms is more likely, but not certain. Also called disease-causing mutation, pathogenic variant, predisposing mutation, and susceptibility gene mutation. 